\title{
Symmetrized dot pattern analysis for the unsteady vibration state in a Sirocco fan unit
}

José González, Jesús M. Fernández-Oro, Laura Delgado, Katia Ma argüelles, David Rodríguez, Sandra Velarde-Suárez, David Méndez

Universidad de Oviedo, Área de Mecánica de Fluidos.

Campus de Viesques. c/WifredoRicart s/n. 33203 Gijón, Asturias (Spain).

Corresponding author: José González. E-mail: aviados@uniovi.es

\begin{abstract}
Sirocco fan units, also known as squirrel cage fans, are usually installed at the air conditioning systems of vehicles used in public transport. They must provide an accurate ventilation flow rate, with high efficiency and performance, but also without operative problems like underperforming, outages or breakdowns, which usually lead to compromise the comfort and quietness of the passengers. Due to their heavy duty, they are susceptible of such problems which finally cause poor ventilation, magnified in highly crowded situations, and important noise generation with uncomfortable vibrations. Periodic maintenance and regular monitoring are thus essential to control that the fan operative range ensures a correct operation.

This paper deals with a series of working failures, typically encountered in industrial applications with Sirocco fans, like impeller unbalances, obstructed channels or blocked inlets. A database with vibration signals has been measured experimentally to obtain the forced response of the fan as a function of the flow rate for different troubled scenarios. Following, a Symmetrized Dot Pattern (SDP) technique, that transforms the temporal waveforms into polar maps, is employed to generate dot patterns which can discriminate between different working conditions and failures, allowing an easy visual identification. The final objective is to show the potentiality of this technique as a fault diagnosis methodology for typical squirrel cage fans, providing optimal parameters for its use. The results obtained are also compared with other diagnosis techniques, previously employed by the authors, for a complementary analysis of the vibrating condition of these centrifugal fans.
\end{abstract}

Keywords: Signal Processing. Maintenance. Tonal noise. Turbomachinery flows. Sirocco Fans, Symmetrized Dot Pattern (SDP) technique. 


\section{INTRODUCTION}

Sirocco fan units, also being referred to in the literature as squirrel cage fans, are centrifugal turbomachines with forward curved blades. They are typically manufactured in rather small sizes and are widely used in a variety of applications like industrial plant applications, kitchen range hoods and many others. Of particular interest for the present study, they are quite widespread on transportation applications, as in buses or trains. Some of the important geometrical features, as the short chord and the large number of blades, were previously pointed out by Kind and Tobin [1], where a particular and detailed study of the geometry and flow implications is given. As stated by Cau et al. [2], the flow structure is globally far from being well known, and with part of the operation ranges within the limits of the performance curve, thus promoting flow separation on the blades.

Jardine et al. [3] have shown most of the possible options when dealing with different maintenance protocols and frequency analysis oriented towards the condition-based maintenance. The Fast Fourier Transform (FFT), in the framework of rotating machinery, has become a standard method to obtain the corresponding spectra revealing its composition in the frequency domain, as stated by Shi [4] and Shi et al [5]. Nevertheless, and opening the practical options, some other current methods are available in the literature, see for instance Höss et al [6]. Also, with regard to the useful life and maintenance for fans, the article by Wang et al. [7] proposes a theoretical model for the fans degradation prediction.

In what may be regarded as the first step of the present article, González et al [8] have shown a feasible option by using a numerical approach together with a Purified Orbit Diagram (POD) technique. The POD technique consists in the spectral analysis of time signals that are forward post-processed to preserve the relevant frequencies only, i.e., the values with higher amplitudes at a set of discrete frequencies. The so-called purified spectrum is thus obtained from the different temporal signals analyzed, which are finally employed to construct an orbit diagram. This diagram is composed through the vector summation of horizontal and vertical components, so horizontal and vertical vibration signals have to be recorded $[4,5]$. The mathematical equations to be considered to obtain both horizontal and vertical components of the orbit diagram are given in the previously mentioned reference [8].

As a natural extent, the study presented here describes a complementary methodology for predictive maintenance that handles the fan operating diagnosis using a visual technique, the socalled Symmetrized Dot Pattern (SDP). This SDP technique shows visually the amplitude and frequency variations of pressure or vibration signals, rendering a SDP diagram in a polar graph. Consequently, the visual maps obtained, which resemble the operative conditions of the system or machine for a particular situation, can be compared with the maps of the baseline working operation for diagnosis. In this context, the technique allows to determine if a machine is performing properly and, if it were not the case, to identify the corresponding failure through image matching. Additionally, the technique has shown its ability to provide accurate fault diagnosis even during reduced acquisition times (a limited number of rotor revolutions), thus enabling its fast application to a real-time monitoring (Sheard et al. [9]).

The SDP technique was firstly introduced by Pickover [10] for the visual characterization of speech waveforms in automatic human-voice-recognition algorithms. Since the SDP pattern provides a local visual correlation that can be applied to detect the significant features of any signal, other researchers have taken advantage of this technique for signal monitoring of rotating machinery. More precisely, Shirai et al. [11] were the first to apply this method to detect irregularities in the rotational parts of a filter fan analyzing audio signals, extended lately by Shibata et al. [12]. The technique has been also applied for IC engines by Wu and Chuang [13], analyzing both microphone and accelerometer signals in the drive axle shaft and in the cooling 
centrifugal fan of a four-cylinder diesel engine. The detection of aerodynamic noise sources has been also explored using this methodology (Bianchi et al. [14]), conducting a qualitative evaluation of the differences in noise emitted from the different impellers of axial fans. Later, Sheard et al. [9] explored the possibility to define a stall-detection methodology for low-speed axial-flow fans based on an SDP database capable to differentiate between stall conditions. Further discussion on the topic was presented by Bianchi at al. [15] afterwards, promoting this signal visualization technique for in-service stall detection in industrial fans. More recently, Xu et al. have employed the technique over a centrifugal fan, analyzing both accelerometer signals in the rotating shaft [16] and pressure signals in the fan casing [17], providing also an image matching algorithm for the fault diagnosis of the machine.

In the present paper, the SDP technique is extensively applied in a Sirocco fan unit, obtaining the visual patterns of vibration signals which have been measured with accelerometers in both horizontal and vertical positions of the casing. Baseline operation, as well as two additional scenarios with given faults, namely a rotor imbalance and one blocked passage have been considered for the measurements. The results reveal that the SDP technique is capable to discriminate between the different failures, and even between operating flow rates, in case that an accurate selection of the parameters involved in the SDP transformation is done. The SDP technique allows the detection of incipient fan failures, preluding a maintenance protocol for small centrifugal fans, and ensures a real-time monitoring of the fan working conditions in an easy way with an extremely reduced data requirement.

\section{FAN GEOMETRICAL ARRANGEMENTS}

The Sirocco fan studied in this article is a double impeller unit, arranged as shown in Fig. 1. The twin impellers are placed at both sides of the electrical DC motor and are supported in a cantilever arrangement by the two sides of the shaft. The blades define a staggered configuration with two semi-impellers, in order to optimize the flow quality. The shaft promotes the rotation of both impellers in a forward direction. In such arrangement, there is a free inlet, at each impeller side opposite to the placement of the motor and a so-called obstructed inlet, at the motor side of each impeller. Therefore, each semi-impeller cannot produce a totally symmetric flow pattern because the boundary conditions are non-symmetric. Nevertheless, considered as a whole, the two twin impellers work within a symmetric geometrical arrangement. From the manufacturing point of view, each impeller is built with two parts, which are linked together by a central plate at the midspan, as can be observed in Fig. 1.

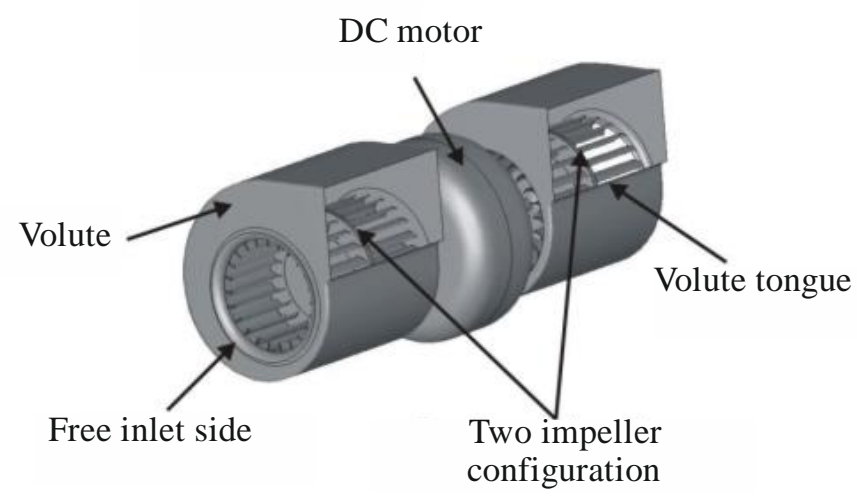

Figure 1.- Simplified sketch of the Sirocco fan studied. 
The semi-impellers are composed of 23 blades, circumferentially staggered to minimize the mechanical interactions (see Fig. 2, left). The total width of the whole impeller is $94 \mathrm{~mm}$. As external casing for the whole unit, there is a volute that diffuses the flow towards two rectangular outlet sections (as can be observed in Fig. 2, right).
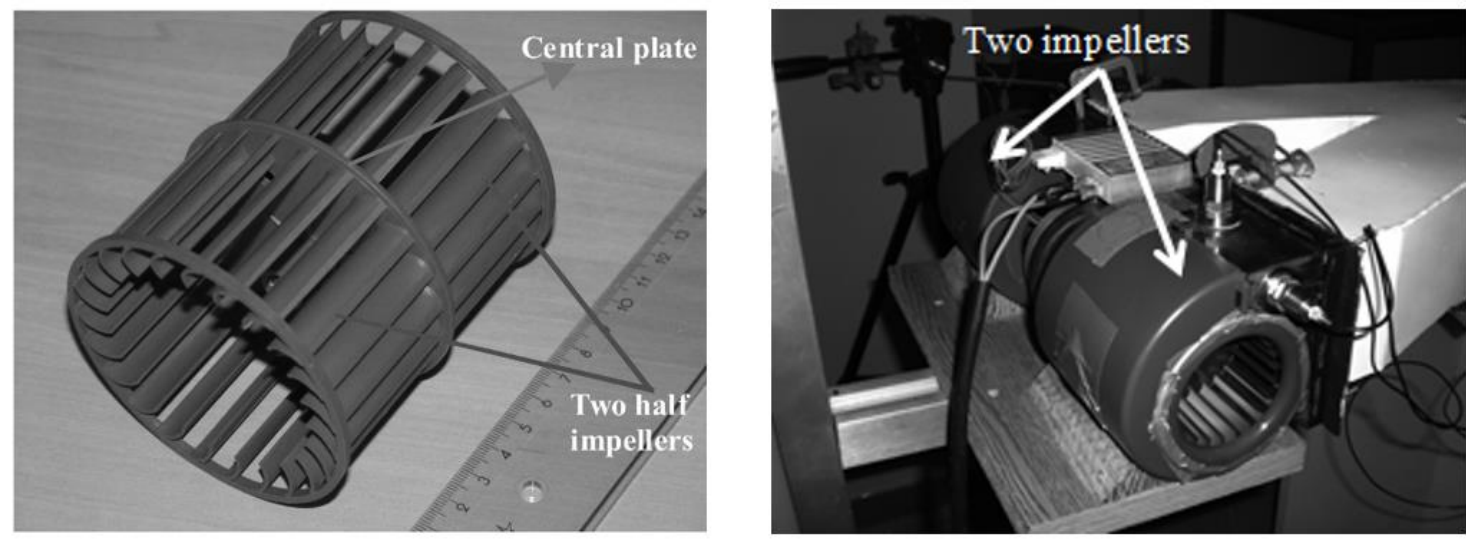

Figure 2.- Each of the twin impellers (left) and fan installed in the bench during the tests (right).

\section{EXPERIMENTAL SET-UP DESCRIPTION AND MEASUREMENTS}

With the fault diagnosis protocol in mind, experimental measurements were taken for different working conditions of the fan, introducing typical failures encountered during its regular operation. The obtained measurements have provided a comparison of the fans performance between baseline and fault operation. The facility, which was properly instrumented to carry out the designed measurements, has been arranged according to the guidelines offered in ISO Standards [18] (this standard is in agreement with the British Standard used for the building up of the test bench, BS 848-1:1997, "Fans for general purposes. Performance testing using standardized airways" and also in the Spanish Standard UNE 100214:1990). The fan is placed at the initial section of the pipe and with a regulation cone installed in the discharging region (see Fig. 3). The performance curve is also available and discussed in detail in [8].

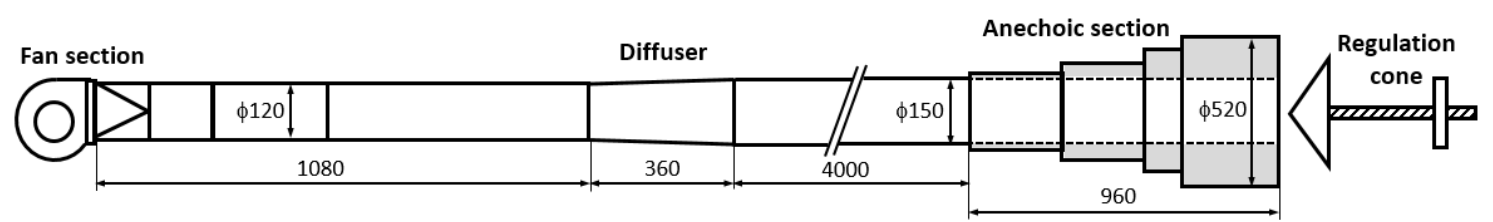

(All Dimensions are in $\mathrm{mm}$ )

Figure 3.- Experimental facility for the tests (according to BS 848-1:1997).

For the present study, the main variable is the vibrating state of the fan, which is monitored using two B\&K-4384 fast response piezoelectric accelerometers (see Fig. 2, right). They are connected to a charge amplifier, B\&K-2635, especially suitable for acceleration measurements. The sensitivity of the sensors is $10 \mathrm{pC} / \mathrm{g}$. The experimental cut frequency for the accelerometers is 10 $\mathrm{kHz}$, although, and due to the practical arrangement on the set-up, the maximum valid frequency is considered to be close to $3.0 \mathrm{kHz}$. This value is well over the mechanical blade passing frequency, which is of interest for the study, obtained to be at maximum $2.0 \mathrm{kHz}$ (corresponding to rotating velocities up to $5000 \mathrm{rpm}$ ). 
The whole set-up is schematically represented in Fig. 4. The measurements chain is completed with the acquisition unit Harmonie, connected to the PC. Values below 2\% for the final SDP data were found when repeatability tests were carried out. Two sensors have been placed in the volute of the fan (Fig. 4). Additional values for the geometrical arrangement and bench definition have been already published in González et al. [8].

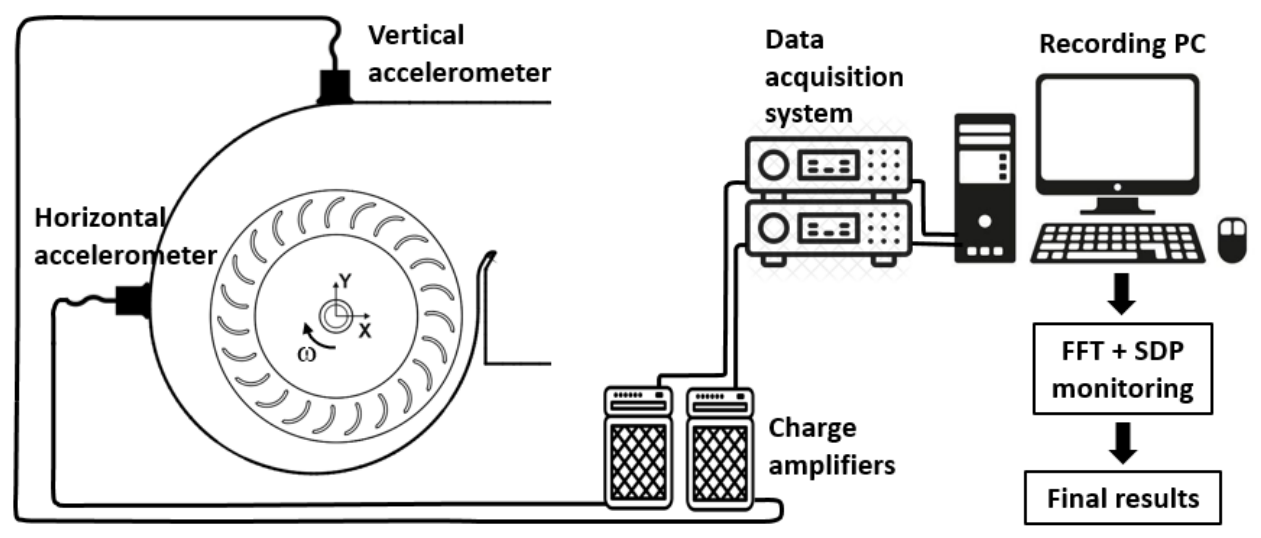

Figure 4.- Measurements chain for the SDP technique.

To account for the influence of the fan working point on the SDP values, three different flow rates were studied, according to the values shown in Table 1 for a rotating speed of $3600 \mathrm{rpm}$. The medium flow in Table 1 corresponds to the nominal point of the present fan $\left(Q=0.098 \mathrm{~m}^{3} / \mathrm{s}\right)$.

\begin{tabular}{|l|c|}
\hline \multicolumn{1}{|c|}{ Considered flow rate } & Flow rate coefficient, $\phi(-)$ \\
\hline Low flow & 0.21 \\
\hline Medium flow & 0.57 \\
\hline High flow & 0.88 \\
\hline
\end{tabular}

Table 1.- Different flow rates considered in the study.

Additionally, three operating conditions have been considered for the experimental database: the normal one (baseline operation), obstructed channel and unbalanced impeller operation. In this investigation, the impeller arrangement for these failures is shown in Fig. 5. A detail of the obstructed channel failure is shown in Fig. 5-left. For the unbalance of the impeller, small metal pieces of $1.6 \mathrm{~g}$ (including the fixing material) were placed upon the fan blades, see Fig. 5-right. It must be noted that the case with the blocked inlet changes the performance operation of the fan as well as the working flow rates, so a direct comparison with the normal operation becomes unrealistic in terms of flow rate.
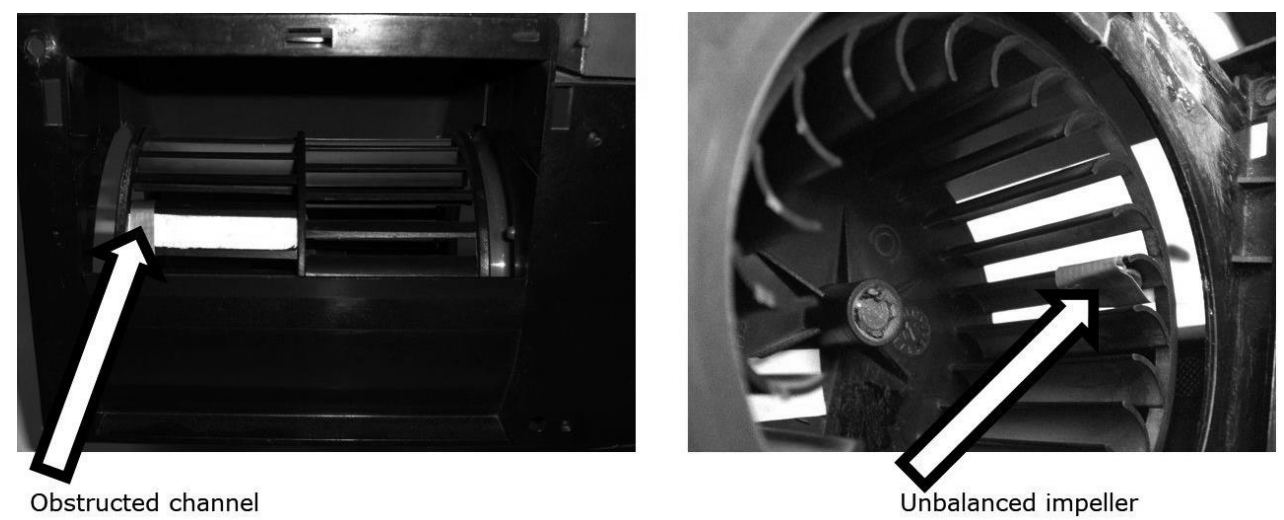

Figure 5.- Impeller modifications for the failure diagnosis. 
As a first approach, maximum accelerations obtained with the purified orbit diagrams (as discussed in [8], combining the measurements of both sensors unsteadily in a Euclidean norm) are provided in Fig. 6 for the three considered flow rates as a function of the working condition. A filtered FFT preserving the relevant harmonics only is employed to reconstruct horizontal and vertical components, which are then combined to plot the orbital evolution of the acceleration. As a result, typical ellipsoidal shapes are obtained, with a maximum radius that is adopted as the maximum acceleration for each case. Those values, provided by the POD technique described in [8], are revisited and shown here in the figure below as a reference for the SDP technique, in order to present a general background and an order of magnitude for the different failures. As expected, the results for the baseline operation are found to be the lowest ones and particularly showing a decreasing value as a function of the flow rate. In the other cases, for the obstructed channel failure, a maximum vibration state is found for the nominal flow rate, with decreasing values for increasing flow rates; whereas for the unbalanced rotor failure, a progressive decrement of the vibrations is obtained as the flow rate is increased. These simple results seem to indicate that the unbalanced rotor failure promotes higher values of the maximum vibration orbit, except for the higher flow rates, when the obstructed channel failure becomes critical.

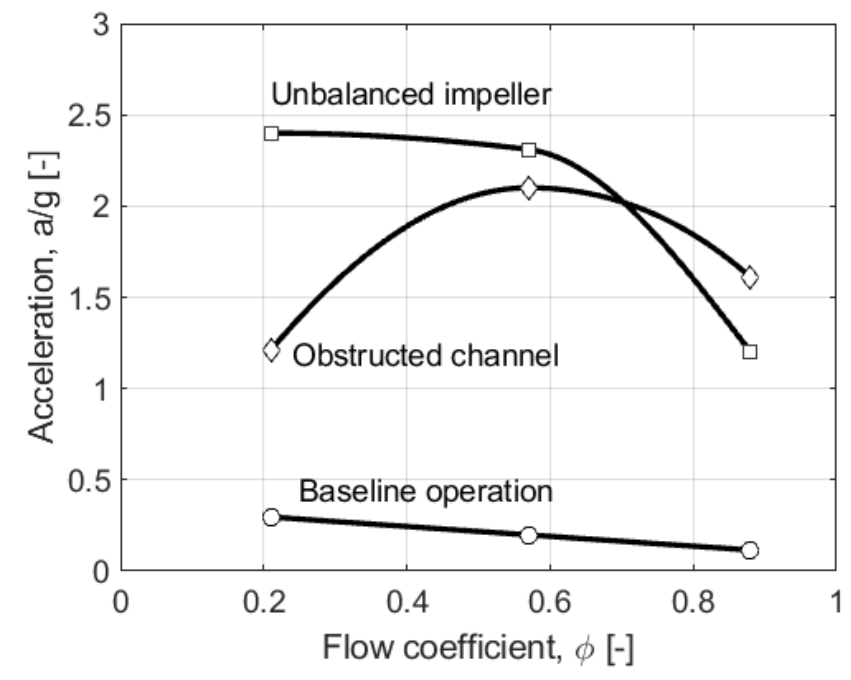

Figure 6.- Maximum accelerations found with the POD analysis.

\section{SDP ANALYSIS}

\section{General background}

The SDP technique was firstly introduced in 1986 by Pickover [10] as an algorithm which maps normalized time waveforms into symmetrized dot space and thus creates a scatter plot of neighbor amplitudes on a polar graph. Basically, SDP involves a transform into a set of dots having mirror symmetry, in which the time waveform of the sound signal is visualized as a snowflakeshaped pattern of (typically) six-fold symmetry (Shibata et al., [12]). Therefore, the method is used to show changes in the amplitude and frequency of a time series signal by means of a visual representation, normally in an easy and understandable way.

In this study, this technique is used to link each vibration signal with its operating condition respectively, so that it allows to assess visually whether a given machine is operating properly and, if it were not the case, to provide the diagnosis of its most probable failure. Because the SDP patterns provide visual correlations with a global perception, it can be potentially employed for the detection and characterization of significant features in sound signals [10]. 


\section{Mathematical framework}

With this technique, a radial and an angular component is firstly assigned to each point in the input signal of the temporal domain. Following, the visual representation is done by mapping these components on a polar basis, generating the so-called symmetrized dot pattern. The data is plotted to be symmetrical about an initial reference line on the polar plot, which is usually selected to be at $60 \mathrm{deg}$. The dot pattern obtained is then repeated six times to cover the whole circumference, obtaining a final characteristic representation with a six-fold symmetry. This angle of rotation should not be made small to avoid that initial and cloned points are overlapping, which makes difficult a useful discrimination between different patterns.

The mathematical expressions that were used to do the polar transformation R(i) from temporal signals to SDP representations are given by:

$$
\left\{\begin{array}{l}
\mathrm{R}(\mathrm{i})=\frac{\sigma(\mathrm{i})-\sigma_{\min }}{\sigma_{\text {max }}-\sigma_{\min }} \\
\Theta^{+}(\mathrm{i})=\Theta_{0}+\frac{\sigma(\mathrm{i}+\mathrm{L})-\sigma_{\min }}{\sigma_{\max }-\sigma_{\min }} \xi \\
\Theta^{-}(\mathrm{i})=\Theta_{0}-\frac{\sigma(\mathrm{i}+\mathrm{L})-\sigma_{\min }}{\sigma_{\max }-\sigma_{\text {min }}} \xi
\end{array}\right.
$$

Where $\mathrm{i}$ is the position of the considered dot (for a total number of points $\mathrm{N}=\mathrm{t} / \Delta \mathrm{t}$, being $\mathrm{t}$ the sampling time and $\Delta t=1 / \mathrm{f}$, where $\mathrm{f}$ is the sampling frequency); $\mathrm{L}$ is the time lag coefficient, $\sigma$ (i) is the value of the vibration data of the considered dot; $\sigma_{\max }$ and $\sigma_{\min }$ respectively represent the maximum and minimum values of the original periodic wave (Fig. 7); $\Theta_{0}$ is the rotation of initial angle of any reference line; $\xi$ is the gain of the plotting and $\Theta^{+}$and $\Theta^{-}$are the angles in the polar diagram. For the present database, only the positive angles $\Theta^{+}$have been used for the representation.
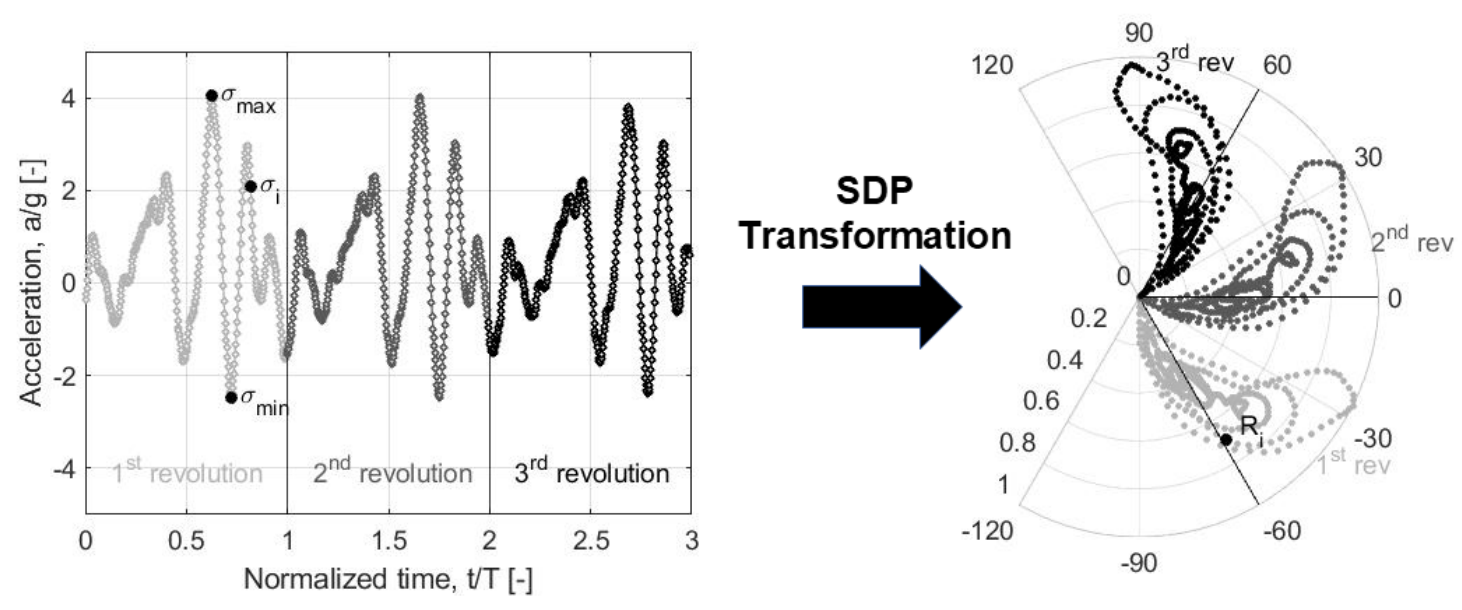

Figure 7.- Typical time signal with relevant points for SDP technique (left) and corresponding SDP points after transformation (right). 
The curvature and the fuzziness of the different lobes composing the SDP diagrams are severely conditioned by the selection of the parameters introduced in the definition (i.e., the lag coefficient and the gain). For this reason, the use of the technique as a method for fault diagnosis, capable to identify different phenomena in rotating machinery, depends on the choice of value for time lag and angular gain. Shibata et al. [12] proved that the selection of the values of the time lag $(\mathrm{L})$ and the angular gain $(\xi)$ depends significantly on the research object. Pioneering works by Pickover [10] for human voice recognition employed a time lag equal to unity, firstly envisaging that a characteristic pattern emerges only when the autocorrelation has a certain lag. Moreover, De Rosier et al. [20] established that there is not an appropriate way to choose the best option, so trial and error must be used to find optimal selections although they suggested a value between 6 and 10 to obtain a stable pattern. Additionally, Bianchi et al. [14] also determined that, because of the lack of established methodologies in the extant literature for the determination of the appropriate SDP parameterization, lag and gain values are to be chosen using a pragmatic trial-and-error approach.

\section{Literature survey}

In order to select an appropriate value for both $\mathrm{L}$ and $\xi$ parameters, a literature survey concerning the application of this methodology for the diagnosis of signals in rotating machinery was firstly performed. Table 2 shows the typical ranges found, as well as additional information summarizing typical acquisition frequencies, types of signals analyzed, and machines considered.

\begin{tabular}{|c|c|c|c|c|c|c|c|c|}
\hline Reference & Year & Machine & $\begin{array}{c}\omega \\
(\mathbf{r p m})\end{array}$ & $\begin{array}{c}\text { Fad } \\
(\mathrm{kHz})\end{array}$ & $\begin{array}{c}\text { Tad } \\
(\mathbf{s})\end{array}$ & Sensors & $\mathbf{L}$ & $\xi$ \\
\hline $\begin{array}{l}\text { Shibata et } \\
\text { al. [12] }\end{array}$ & 2000 & HVAC fan & $\mathrm{N} / \mathrm{A}$ & 20 & $\mathrm{~N} / \mathrm{A}$ & $\begin{array}{l}\text { Accelerometers } \\
+ \text { Microphones }\end{array}$ & 5 to 10 & $\begin{array}{l}20 \text { to } \\
40\end{array}$ \\
\hline $\begin{array}{l}\text { Wu and } \\
\text { Chuang [13] }\end{array}$ & 2005 & IC Engine & $\begin{array}{l}750 \text { to } \\
3000\end{array}$ & $\mathrm{~N} / \mathrm{A}$ & $\mathrm{N} / \mathrm{A}$ & $\begin{array}{l}\text { Microphones + } \\
\text { Accelerometers }\end{array}$ & $\mathrm{N} / \mathrm{A}$ & $\mathrm{N} / \mathrm{A}$ \\
\hline $\begin{array}{l}\text { Wu and } \\
\text { Chuang [13] }\end{array}$ & 2005 & $\begin{array}{l}\text { Centrifugal } \\
\text { fan }\end{array}$ & $\mathrm{N} / \mathrm{A}$ & $\mathrm{N} / \mathrm{A}$ & $\mathrm{N} / \mathrm{A}$ & Microphones & $\mathrm{N} / \mathrm{A}$ & $\mathrm{N} / \mathrm{A}$ \\
\hline $\begin{array}{l}\text { Hameed } \\
\text { and Samson } \\
\text { [19] }\end{array}$ & 2009 & $\begin{array}{l}\text { Ball } \\
\text { Bearings }\end{array}$ & $\begin{array}{l}500 \text { to } \\
1500\end{array}$ & 20 & $\mathrm{~N} / \mathrm{A}$ & Accelerometers & 5 to 10 & $\begin{array}{ll}20 & \text { to } \\
40 & \end{array}$ \\
\hline $\begin{array}{l}\text { Bianchi et } \\
\text { al. [14] }\end{array}$ & 2009 & $\begin{array}{l}\text { Low-speed } \\
\text { axial fan }\end{array}$ & 950 & 10 to 20 & 60 & Microphones & 10 & 20 \\
\hline $\begin{array}{l}\text { Sheard at al. } \\
\text { [9] }\end{array}$ & 2011 & $\begin{array}{l}\text { Low-speed } \\
\text { axial fan }\end{array}$ & 1480 & 50 & 10 & $\begin{array}{l}\text { Flush-mounted } \\
\text { pressure } \\
\text { sensors }\end{array}$ & 7 to 30 & 1 to 20 \\
\hline $\begin{array}{l}\text { Bianchi et } \\
\text { al. [15] }\end{array}$ & 2013 & $\begin{array}{l}\text { Low-speed } \\
\text { axial fan }\end{array}$ & $\begin{array}{l}930 \text { to } \\
950\end{array}$ & 10 & 60 & Microphones & 1 to 50 & 1 to 50 \\
\hline $\begin{array}{l}\mathrm{Xu} \text { et al. } \\
{[16]}\end{array}$ & 2016 & $\begin{array}{l}\text { Centrifugal } \\
\text { fan }\end{array}$ & 1200 & 1.6 & $\mathrm{~N} / \mathrm{A}$ & Accelerometers & 1 to 10 & $\begin{array}{ll}20 & \text { to } \\
40\end{array}$ \\
\hline $\begin{array}{l}\text { Xu et al. } \\
{[17]}\end{array}$ & 2017 & $\begin{array}{l}\text { Centrifugal } \\
\text { fan }\end{array}$ & 1300 & 0.32 & 1 & $\begin{array}{l}\text { Pressure } \\
\text { sensors }\end{array}$ & 5 to 15 & $\begin{array}{ll}10 & \text { to } \\
30\end{array}$ \\
\hline
\end{tabular}

Table 2. Typical parameters employed in the literature for the application of the SDP technique in the vibration analysis of rotating machinery.

In the case of rotating environments, typical ranges between 1 and 30 have been used as lag coefficients by a wide number of researchers. Complementarily, angular gains in the range between 1 and 40 deg are also been reported in several investigations. Recent investigations by Xu et al. $[16,17]$ seem to indicate that the angular gain must be reduced as the rotational speed is progressively increased. 


\section{Sensitivity analysis}

The use of sine-like test signals to check the performance of the SDP representation and judge its convenience for the analysis of the parametrization has been employed with good results for several authors in the past ([10-14]). It was observed that, in general, the higher the frequency of the signal, the more unclear is the shape of the pattern obtained by the SDP method [12]. Besides, it is known that the frequency content of the time waveform manifests in the dot pattern curvature, while the signal variation results in the dot fuzziness, which increase the SDP footprint [9]. The latter feature, correlated with the highest point in the recorded waveform, reflects in the outer radial positions of the dot collection. In summary, it was noticed that the lobe curvature depends on the variety of frequencies of the sampling and the fuzziness on the irregularities of the signals which increases the thickness of the SDP fingerprint.

On the other hand, sensitivity analysis performed over real acquired signals have evidenced that (1) the pattern arms are gradually losing their curvature when low frequencies are predominant in the time series, and (2) that the footprint in the SDP maps extends when the instability in the original signal is increased (higher fluctuations). Moreover, it was observed that the sampling rate is not a critical parameter, so it is possible to obtain the same signal interpretation operating at a lower sample rate.

By analyzing the vibration signals by the SDP technique and observing the SDP patterns, it was found that the discrimination among the SDP patterns of vibration signals under different operation states can be optimal by setting $L=6$ and $\xi=1.2 \mathrm{deg}$, thus producing the best SDP fingerprints for our particular database. This selection has been supported by an area-based criterion, which fixed a prescribed size for the lobes in order to ease their identification. In particular, a range between $25 \%$ and $50 \%$ of the available area in the plot was considered sufficiently accurate to guarantee the patterns recognition. Figure 8 shows such variations, for an experimental test signal (nominal conditions at a higher flow rate), depending on the values of the time lag and the angular gain. Note that these values are also aligned with typical ranges given in Table 2. The high rotational speed of the sirocco fan justifies the severe reduction in the value of the angular gain with respect the values found in the literature.
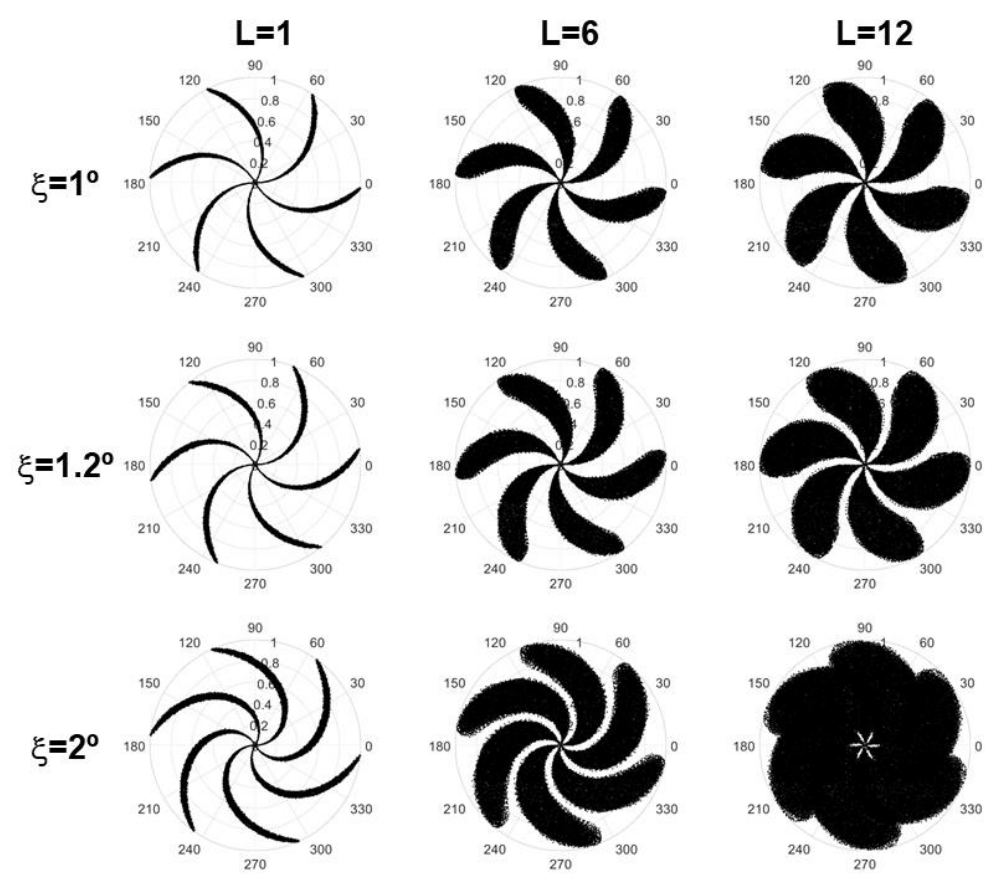

Figure 8.- Parameters sensitivity for the SDP analysis. 
The sampling frequency and the acquisition time were of $25.6 \mathrm{kHz}$ and $15 \mathrm{~s}$ respectively. These values allow determining the number of rotor revolutions during the sampling time, which depends on the fan flow rate for this type of Sirocco fans. In the case of medium flow rate, for a characteristic rotational speed of the rotor around $4500 \mathrm{rpm}$, the sampling time lasted 1125 revolutions of the impeller.

Sheard et al. [9] revealed that further reductions of the sampling frequency showed no relevant impact on the representations, producing just a downscaling of the SDP diagrams. This means that, although a sampling rate of $25.6 \mathrm{kHz}$ was employed in this study, it would seem to be possible to obtain the same signal interpretation operating at a lower sample rate. With respect the total sampling time, they also found that short sampling time durations had a very reduced influence on their results. Even time windows comprising roughly one revolution of the machine were sufficient to obtain well-discriminated SDP maps. This guideline has been checked for the present database rendering the SDP representation of the vibration signal in case of medium flow rate for nominal conditions, using the total acquisition time or reduced periods with one-tenth and one-hundredth of the original time window. The results, shown in Figure 9, confirm the marginal influence of this parameter in the SDP technique and explains why this data is absent in most of the references in Table 2.

15 seconds (1100 revs)

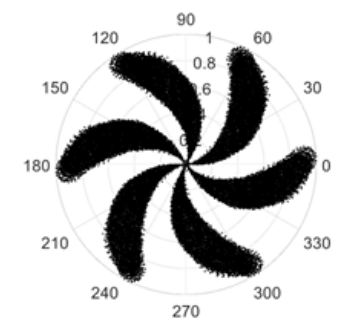

1.5 seconds (110 revs)
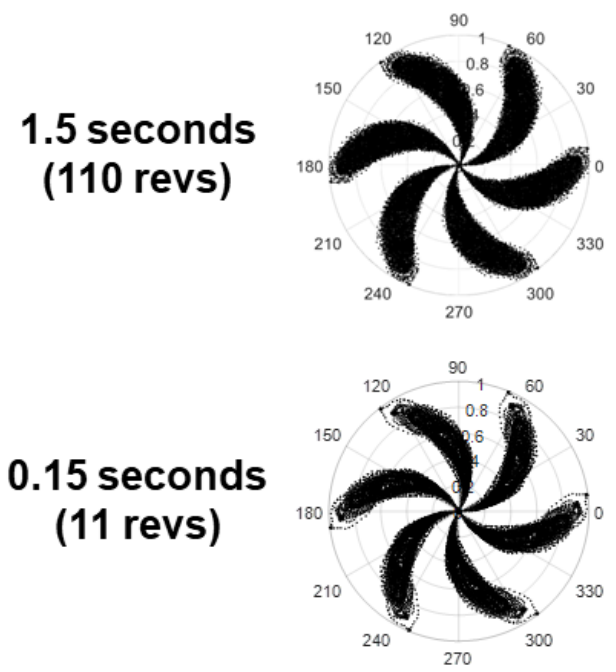

Figure 9.- SDP patterns for different acquisition time (rotor revolutions).

\section{RESULTS AND DISCUSSION}

From the considered failures and flow rates, nine graphs for each one of the accelerometers are to be obtained. The results are shown in Figs. 10 and 11, and will be studied thoroughly in what follows, with special attention to the details allowing to differentiate between the patterns of the studied operations and the corresponding flow rates. More precisely, the differences and their tendencies are discussed in order to obtain general rules to identify the operating condition of the fan. 


\section{Horizontal sensor}

Normal operating (baseline) versus unbalanced rotor: As can be easily observed in the comparison, a quite different shape of the maps is obtained. For the three analyzed flow rates a more bended and thicker map is found for the unbalanced impeller (more irregular). The differences are higher for the higher flow rate, which shows a higher dot fuzziness that makes the SDP fingerprint wider and presents a characteristic region without dots in the outer half of the lobes.

Normal operation (baseline) versus obstructed channel: The obstructed channel SDP fingerprints are wider and more irregular again than the normal SDP fingerprints, with even a characteristic cutting-edge shape at higher flow rates. This bigger dot dispersion shows that the obstructed channel fan vibration is higher with respect to the normal operating condition. Some characteristic regions without dots are found in the inner half of the lobes.

Unbalanced rotor versus obstructed channel: Differences are subtler between the failures, especially at nominal flow rate. However, at low flow rate, the regions with spare dots are higher for the obstructed channel operation, while for the high flow rate, these regions are observed for the unbalanced impeller only. At the nominal flow rate, differences come not so much due to the dot density, but due to the shape of the maps. Again, the unbalanced impeller shows higher bend of the fingerprints.
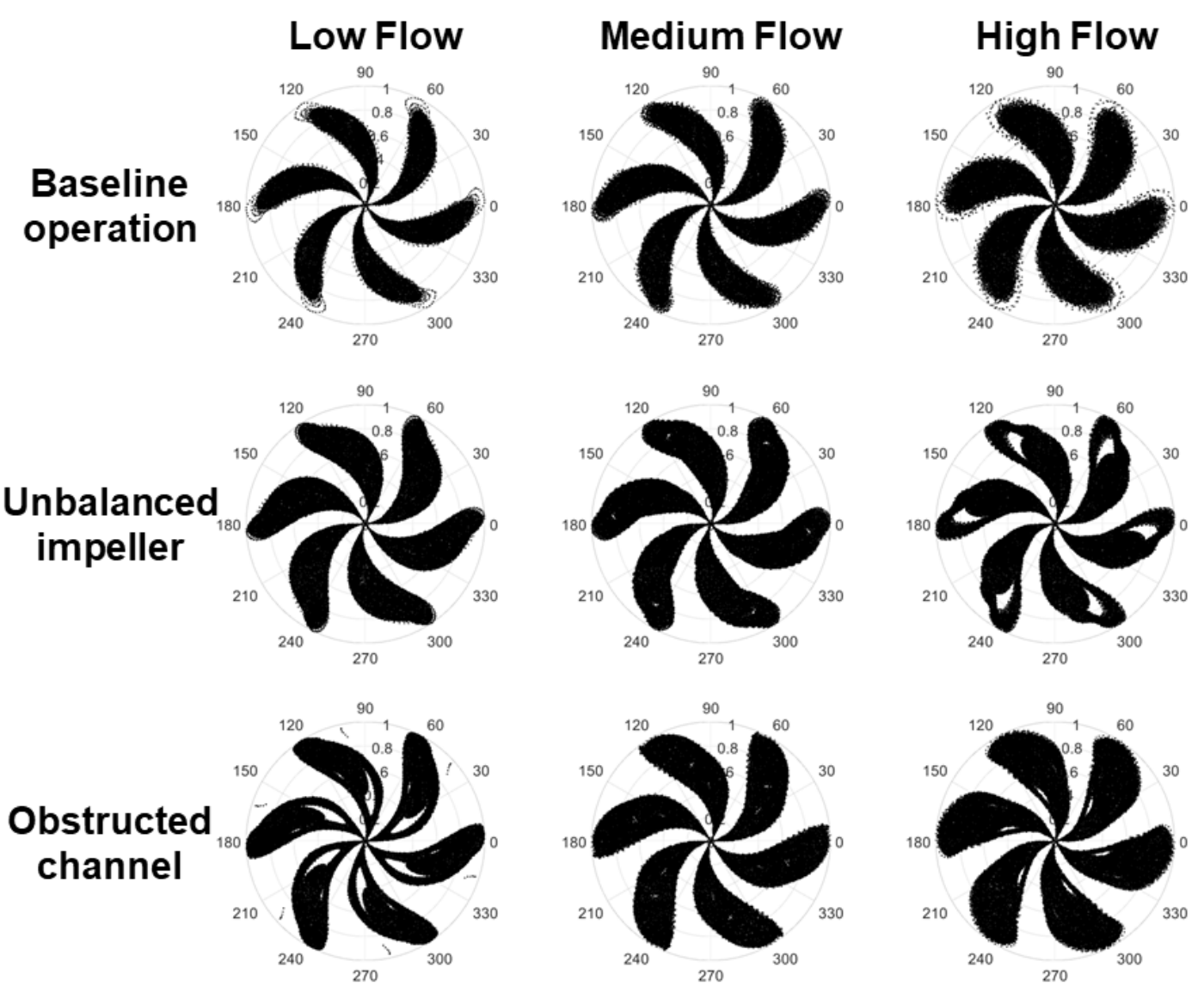

Figure 10.- SDP maps for the horizontal sensor data. 


\section{Vertical sensor}

The results from the vertical sensor (Fig. 11) complement the observed differences. For this sensor, the shape and density of points show more differences than for the horizontal sensor. Globally speaking, the lobes for this sensor show thinner shapes than the horizontal ones.

Normal operation versus unbalanced rotor: Again, the lobes are less bended in the normal operation than in the unbalanced impeller. A bigger difference is found in the lobes density of points, with quite high void regions in the unbalanced impeller operation (for all flow rate conditions).

Normal operation versus obstructed channel: Lobes are thinner for the obstructed channel operation. About the dots distribution, also, quite high regions with no or scarce points are clearly different comparing both operations. Baseline operation shows quite denser and wider lobes. Note also that the fingerprints of the obstructed case for low and high flow rates are supplementary (much like a photographic negative).

Unbalanced rotor versus obstructed channel: Like the horizontal sensor SDPs, fingerprints obtained from the vertical sensor visually quite similar between the two types of failures. Regarding the shape, the lobes are more bended for the unbalanced impeller operation. The spare points characteristic regions are placed at different locations depending on the working condition and flow rate. At off-design flow rates, the density of points is higher for the obstructed channel operation, while for the nominal or medium flow rate, both fingerprints show more or less the same density.
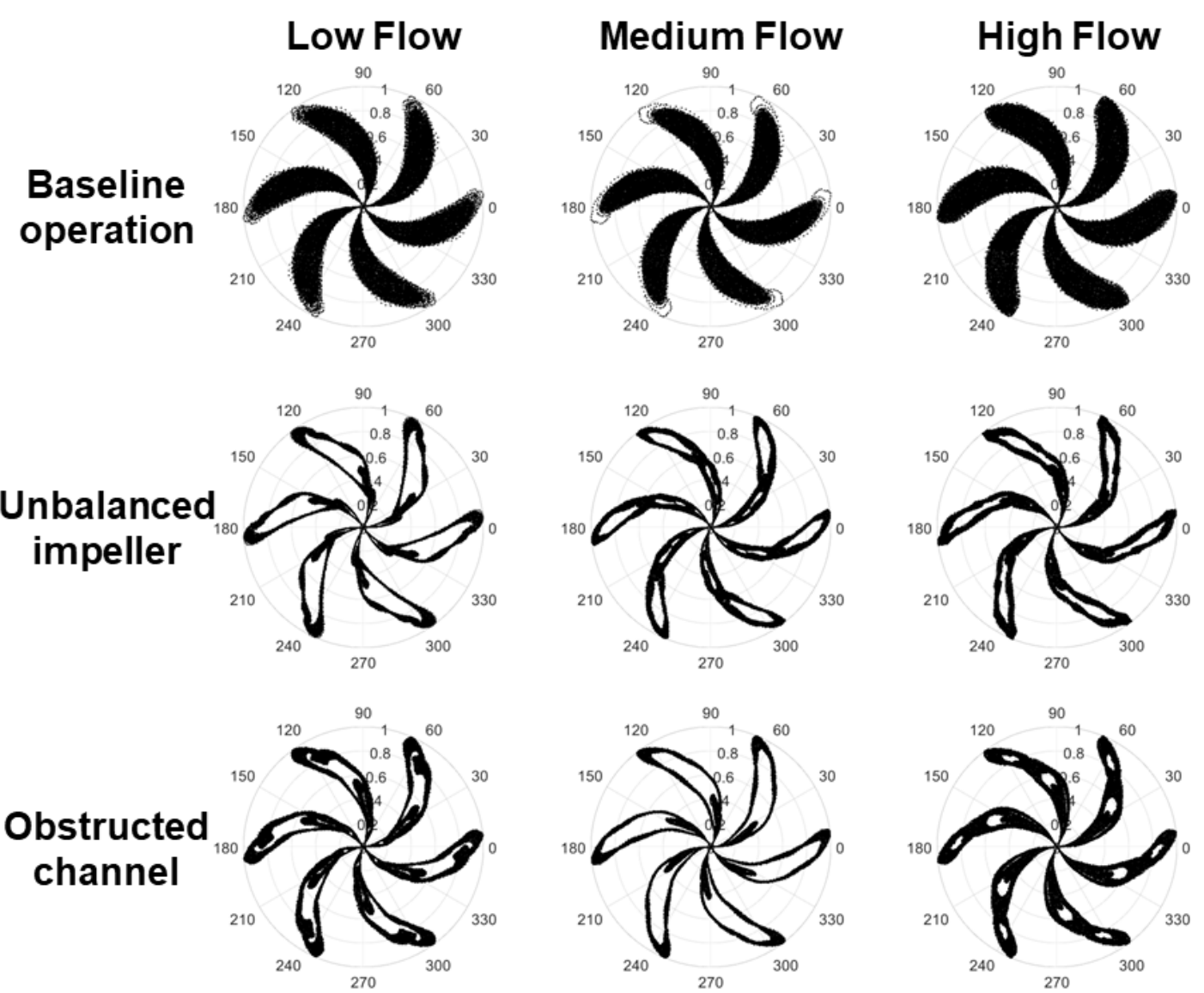

Figure 11.- SDP maps for the vertical sensor data. 


\section{Modified SDP}

Another possibility of interpretation would be to eliminate of the SDP fingerprint the external boundaries that encircle the regions without dots. Fig. 12 shows the SDP diagram obtained by the application of this procedure using the vertical sensor data. The lobes of the obstructed channel SDP obtained in this way have a lower length than for the normal operating and unbalanced rotor SDP as Fig. 13 clearly shows. This is due to the fact that, for the obstructed channel operating state, the predominant waves are of low frequency and their amplitude is larger than in the other cases. These modified SDPs may provide an alternative way to differentiate the different working condition.

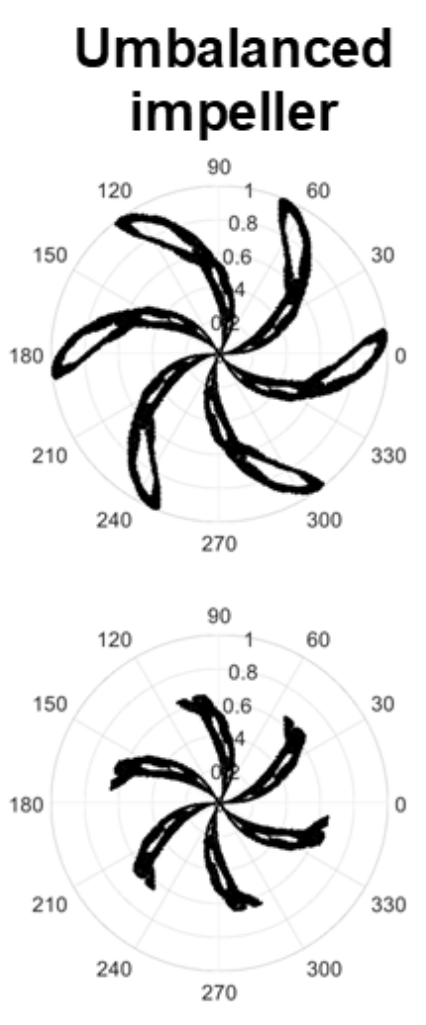

Figure 12.- SDP fingerprint with (top) or without (bottom) external lines.

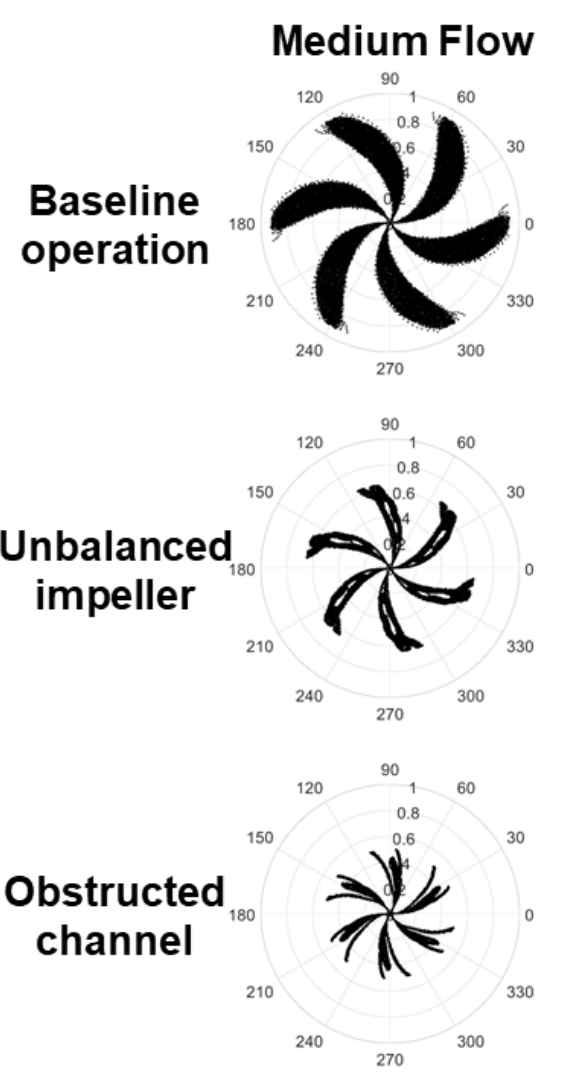

Figure 13.- Modified SPDs for different conditions.

\section{Comparison of SDP and POD techniques}

This final subsection compares the results obtained with the SDP technique for the present investigation with respect to previous results and conclusions derived from a POD analysis of the vibration signals (results of the POD technique taken from González et al., [8] for the same geometry and working conditions). The objective is to show the complementarity of both methodologies, confirming that they are providing coherent guidelines and highlighting general advantages and disadvantages of their use.

Complementing the results of both techniques, the machine operating condition will be determined by the acceleration value and by the orbit shape that shows the direction of the predominant acceleration. As regards SDP fingerprints, the frequency content determines the curvature of the lobes in the patterns, so a major contribution of lower frequencies leads to thinner 
lobes, whereas significant contributions at higher frequencies will result in wider lobes. In other words, the thickness of the lobes is associated to the broadness of the frequency range; Therefore, the larger and more shifted the range with relevant frequencies is, the greater the lobe thickness will be. This observation has been confirmed using a linear regression to correlate the width of the lobes with the normalized mean frequency of the spectra in every fan condition. The mean frequency represents the average contribution of all the frequency range, where each frequency has been weighted by its corresponding amplitude value (i.e., the weighted-average value). The results obtained are shown in Fig. 14, where black dots correspond to the data of the vertical accelerometer, and grey squares stand for the data of the horizontal sensor. Note the excellent correlation found between the variables $\left(\mathrm{R}^{2}=0.91\right)$.

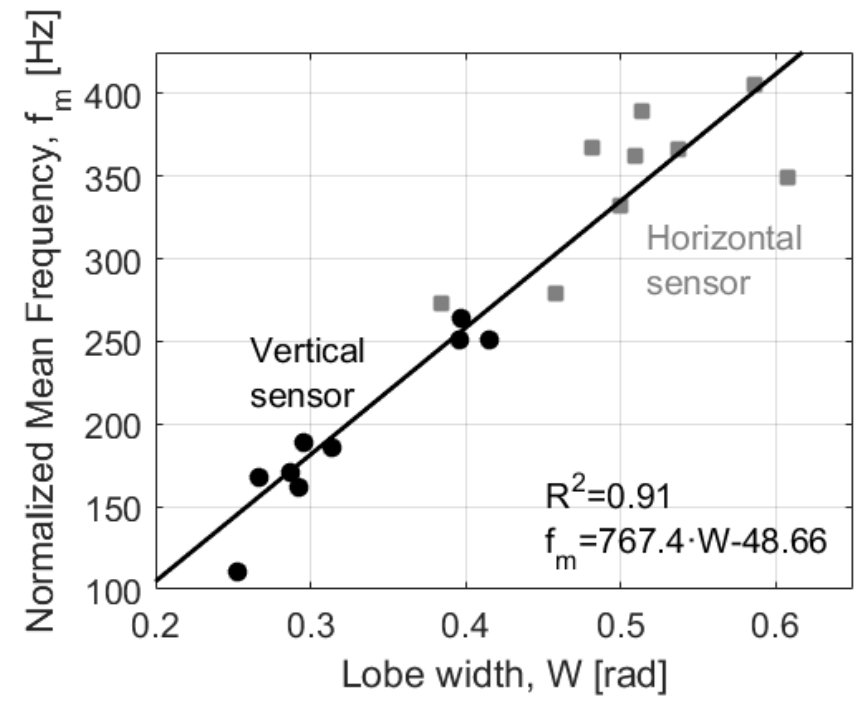

Figure 14.- Linear correlation between the width of the SDP lobes width and dominant frequencies.

Fig. 15 and Fig. 16 show the comparison for the two kind of diagrams for the two off-design conditions, namely, the low flow rate (Fig. 15) and the high flow rate (Fig. 16). A third comparison for the nominal flow rate is available, but no remarkable new conclusions can be drawn from it, so it has been not shown in the article for brevity. In addition to the results from the two mentioned techniques, the FFT amplitudes are also plotted for the different operations, up to a frequency range of $2 \mathrm{kHz}$. Then, each figure is made with three columns, namely the different operations and three rows, that is, the POD technique results (top row), the SDP results for the horizontal sensor (middle row) and the SDP results for the vertical sensor (bottom row). In the case of the SDP, the FFT signal (in terms of Power Spectrum Density or PSD) is also plotted.

For the low flow rate, unbalanced rotor SDP diagram obtained by the vertical sensor data (Fig. 15, bottom row), provides thinner lobes with a big region without dots in its center, which indicates that the predominant waves are of low frequency and of a considerable amplitude. This is reflected by the high acceleration value of the POD diagram, which is much higher for this fault, and also in a bump of the spectrum baseline centered at $200 \mathrm{~Hz}$ approx. (shadowed in grey). Considering the horizontal acceleration value (Fig. 15, middle row maps), the lobe thickness is enlarged, similar to the normal operating lobes, and showing some regions without inner dots because of the higher amplitude waves that are equally distributed in the frequency spectrum. Coherently, the acceleration value of the POD diagram is a little higher than the one for the normal operation, but lower than for the unbalance impeller fault. The bump in the spectrum is now displaced towards a typical value of $400 \mathrm{~Hz}$. 


\section{LOW FLOW RATE}

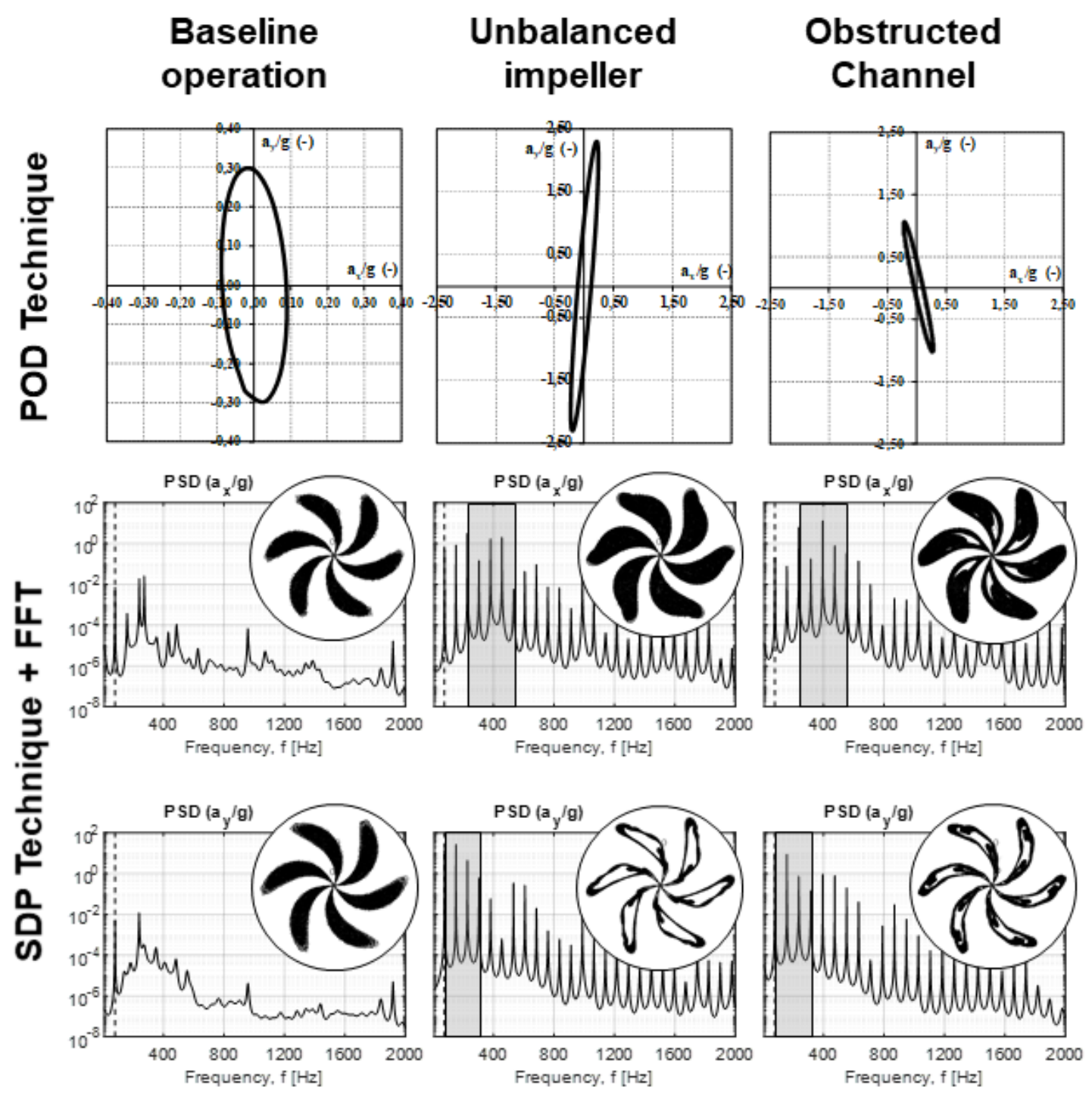

Figure 15.- POD and SDP comparison for the low flow rate.

For the other off-design condition (plotted in Fig. 16) and considering that the POD technique provides higher values for the unbalanced impeller, intermediate for the obstructed channel and lower for the baseline operation, again a direct relationship with the voids in the SDP lobes can be traced. Also, a kind of complementary effect is shown as the difference between the POD values is not so high for the two faults and, for this flow rate, the SDP shows clear differences in both sensors for them. This last conclusion is valid also for the nominal flow rate, though not shown here. 


\section{HIGH FLOW RATE}

\section{Baseline operation \\ Unbalanced impeller}
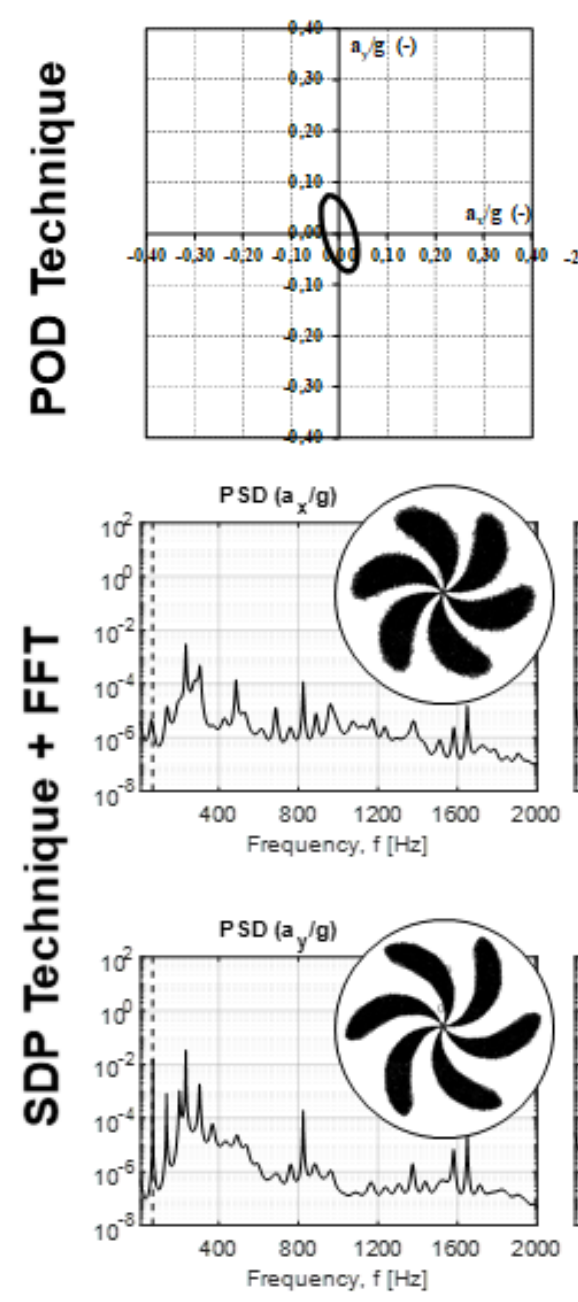
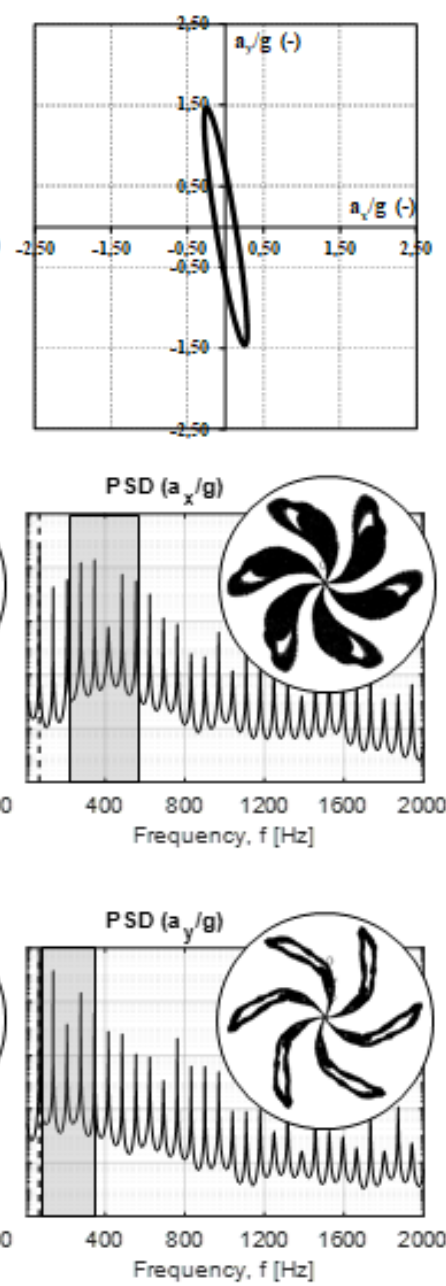

\section{Obstructed Channel}
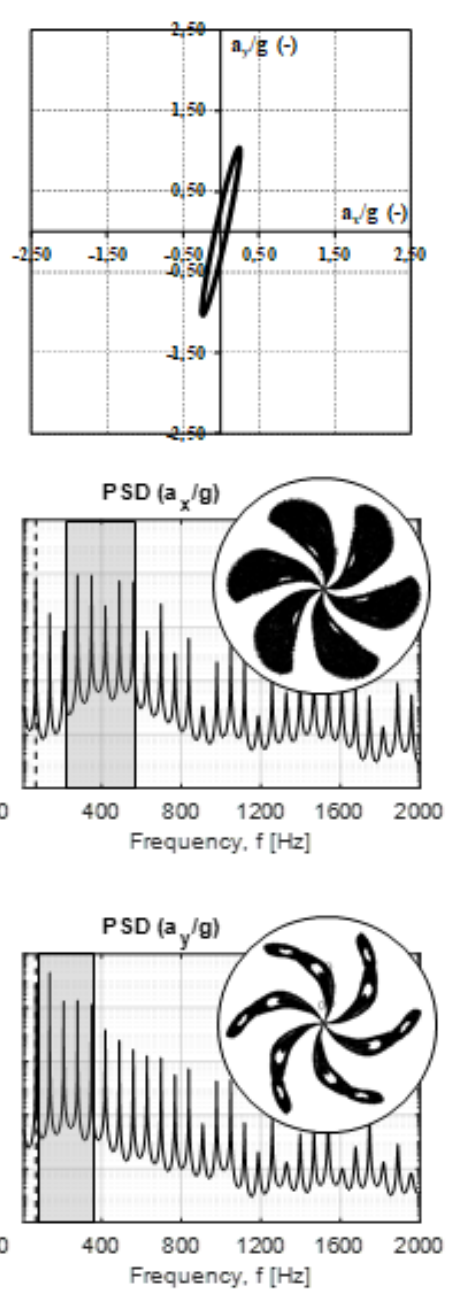

Figure 16.- POD and SDP results for the high flow rate.

After a detailed observation of the results plotted in Figs. 15 and 16, some features of the cross-study of the POD and SDP techniques can be derived. Particularly, the explanation for the presence of holes in the interior of the snowflakes is related to the measured values of the vertical and horizontal vibration acceleration of the fan. That is, high values in the vertical acceleration produce gaps in the lobes of the SDP obtained from the data of the vertical accelerometer. This same criterion is met for the horizontal sensor. When the value of the acceleration is low and the value of the vertical acceleration is similar to that of the horizontal, uniform and completely filled SDPs are produced, which indicates a good operating state. These gaps occur due to the greater difference between the values of the acceleration between two consecutive points of the sample, giving rise to a more unstable operation that makes the points are not evenly distributed in the lobes of the SDP (more instabilities at higher frequencies). In normal operation this does not happen since the values of the acceleration are low and therefore, the variation between the maximum and minimum value is small, allowing all the points to be distributed (broadband spectra) and completely filling the interior of the snowflakes. 
Consequently, as general observations, it can be stated that:

- If high frequencies are dominant in the signals, the lobes are significantly wider. They are much thinner in case of predominant low frequencies. In this squirrel-cage fan, low frequencies are associated to pulsations in the order of the machine perimeter ( $200 \mathrm{~Hz}$ and below), whereas high frequencies range up to $400-600 \mathrm{~Hz}$.

- The external curvature of the lobes is more abrupt and with irregularities if the low frequency content is particularly intense. This is clearly observed at failure conditions for the vertical sensor.

- On the contrary, central (and large) void regions appear if a high frequency is dominant in a spectrum where most of the energy content is distributed at low frequencies (obstructed case at low flow rate for the horizontal sensor).

- When void regions appear in the external zones of the lobes or they are evenly distributed in the interior parts of the lobes, a typical spectrum with descending amplitudes of the harmonic contents is observed (vertical sensor with failure conditions).

- Finally, regular and smooth lobes without void regions are obtained in case of nominal conditions, where broadband spectra without significant harmonics are revealed. This is associated to the fuzziness of the dots distributions, which is more uniform when all-frequency contents are involved in the signals.

\section{CONCLUSIONS}

By the experimental recording of accelerometer signals, a vibration-based analysis of a squirrel cage fan with typical operative failures has been performed. Both horizontal and vertical accelerations in the volute casing of the centrifugal fan have been monitored for two different failures as a function of the flow rate, comparing the results with the baseline operation. An SDP technique has been considered to visualize and characterize the fan operation for a rotor unbalanced and an obstructed channel fault operation.

The SDP technique allows the detection of incipient fan failures. It firstly transforms the temporal waveform of the vibration signals into a polar map of dot patterns that is later used for the visual identification of the vibrational state in the rotating turbomachine. When the SDP fingerprint obtained from the vibration analysis is different from the normal operating SDP, it indicates that the fan is not working properly, and a mechanical failure is expected to overcome in a near future. The application of this methodology requires the selection of optimal parameters (lag coefficient and angular gain) for a correct discrimination of the different operating conditions, as it is shown and conducted in the case of the Sirocco fan units of the present investigation.

The normal operating SDP patterns consist of a kind of snowflake lobes, with evenly distributed dots all over the fingerprint, without void regions and characterized by an evident lack of fuzziness. They also present a tendency to be narrower and more self-concentrated for nominal flow rates, due to the low values of acceleration that appear in that particular case. For the typical failures analyzed, the lobes are more irregular and present several regions without dots inside the SDP fingerprints, denoting the presence of low frequency vibrational components. In addition, the lobes thicknesses and the global fuzziness is significantly increased due to the vibration wave amplitudes caused by the accelerations at higher amplitudes. A linear correlation between the frequency contents and the lobes thicknesses has been successfully confirmed.

The SDP methodology has been also compared to a previous analysis of the authors using a complementary vibration-based technique known as POD. Both procedures have provided similar conclusions and expectations, although the SDP analysis is simpler than the POD technique in what 
refers to its implementation, despite of it requires a deeper qualitative analysis of the results. In addition, the obtained SDP diagrams, with both horizontal and vertical sensors, allow an approximation of the POD orbit through the observation of the lobes shape, considering that its thickness is increased for higher acceleration values. Moreover, the SDP fingerprints are obtained with a simpler data treatment and the search of the characteristic frequencies is even not necessary for the SDP representation because the technique is fully visual. This feature makes the SDP methodology particularly suitable for the real-time monitoring of the fan working conditions. The observed lack of points in the diagrams promote the idea that the denser the SPD is, the closer to the baseline operation the machine is. An explanation was found on the relative importance of the differences between consecutive points in the sampling series promotes such lack of interior points in the observed lobes.

In summary, concerning the declared goals for this research, the SDP technique has shown its ability to allow the detection of incipient fan failures, preluding a maintenance method for small centrifugal fans. The study for fault diagnosis performed here gives a precise idea of the main working range parameters of the SDP methodology and envisages a possible procedure for fault detection in Sirocco fan units within air conditioning systems in vehicles for public transport.

\section{NOMENCLATURE}

a Acceleration, $\left(\mathrm{m} / \mathrm{s}^{2}\right)$.

$\mathrm{D}_{2} \quad$ Impeller diameter, $(\mathrm{m})$.

FFT Fast Fourier Transform, (Amplitude as a function of the frequency).

f Frequency, $(\mathrm{Hz})$.

$\mathrm{f}_{\mathrm{ad}} \quad$ Acquisition frequency, $(\mathrm{Hz})$.

$\mathrm{f}_{\mathrm{m}} \quad$ Normalized mean frequency, $(\mathrm{Hz})$.

g Gravity acceleration, $\left(\mathrm{m} / \mathrm{s}^{2}\right)$.

i Given position of the SDP diagram.

L Time shift (lag coefficient) for the SDP technique, (s).

N Number of data points.

POD Purified Orbit Diagram.

PSD Power Spectrum Density.

Q Flow rate.

$\mathrm{R} \quad$ Polar value of a given signal.

SDP Symmetrized Dot Pattern.

W Lobes width, (rad).

$\sigma \quad$ Value of the vibration signal for the SDP analysis.

$\phi \quad$ Flow rate coefficient, $\phi=\frac{8 \mathrm{Q}}{\pi \omega \mathrm{D}_{2}^{3}},(-)$.

$\xi \quad$ Gain of the values plotted in the SDP diagram.

$\Theta \quad$ Phase values for the SDP analysis, (rad).

$\mathrm{t}$ Time, (s).

$\mathrm{T}_{\mathrm{ad}} \quad$ Acquisition time, (s).

$\mathrm{T}$ Period of one rotor revolution, (s).

$\omega \quad$ Rotating speed, $(\mathrm{rad} / \mathrm{s})$.

$\mathrm{x}, \mathrm{y} \quad$ Horizontal and vertical components. 


\section{ACKNOWLEDGEMENTS}

Firstly, the authors gratefully acknowledge the collaboration and kind permission for the publication of the present material by Internacional Hispacold S.A., with especial mention to Juan Bernal-Cantón. Also, to perform the measurements, it was very valuable the financial support from the "Ministerio de Ciencia e Innovación" under Projects TRA2007-62708, "Tecnologías ecológicas para el transporte Urbano, ecoTRANS" (CDTI), and "Ministerio de Ciencia, Innovación y Universidades" under Project ENE2017-89965-P, "Desarrollo y construcción de turbinas eólicas de eje vertical para entornos urbanos".

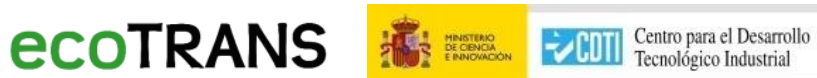

\section{REFERENCES}

[1] Kind, R.J., Tobin, M.G., "Flow in a Centrifugal Fan of the Squirrel Cage Type," ASME J. of Turbomach., 112(1), pp. 84-90, 1990.

[2] Cau, G., Mandas, N., Manfrida, N., Nurzia, F., "Measurements of primary and secondary flows in an industrial forward-curved centrifugal fan," ASME J. Fluids Eng., 109(4), pp. 353-358, 1987.

[3] Jardine, A.K.S., Lin, D., Banjevic, D., "A review on machinery diagnostics and prognostics implementing condition-based maintenance," Journal of Mechanical Systems and Signal Processing, 20 (7), pp. 1483-1510, 2006.

[4] Shi, D.F., "Study on Holodiagnosis for large rotating machinery," Ph.D. Thesis, Xian Jiaotong University, China, 1998.

[5] Shi, D.F., Qu, L.S., Gindy, N.N., "General interpolated Fast Fourier Transform: A new tool for diagnosing large rotating machinery," Journal of Vibration and Acoustics, 127 (4), pp. 351-361, 2005.

[6] Höss, B., Leinhos, D., Fottner, L., "Stall inception in the compressor system of a turbofan engine," ASME J. Turbomach., 122, pp. 32-44, 2000.

[7] Wang, Z-Q., Hu, C-H, Si, X-S, Zio, E., "Remaining useful life prediction of degrading systems subjected to imperfect maintenance: Application to draught fans", Mechanical Systems and Signal Processing, vol. 100, pp. 802-813, 2018.

[8] González, J., Delgado, L., Fernández, J., Velarde, S., Argüelles, K., Rodríguez, D., "Purified orbit diagram and numerical study for a failure analysis of a Sirocco fan", Advances in Mechanical Engineering. Vol. 9(11), pp. 1-17, 2017.

[9] Sheard, A.G., Corsini, A., Bianchi, S., "Stall warning in a low-speed axial fan by visualization of sound signals," ASME Journal of Engineering for Gas Turbines and Power, 133(4), pp. 041601-1 to 041601-10, 2011.

[10] Pickover, C. A., "On the use of Symmetrized Dot Patterns for the visual characterization of speech waveforms and other sampled data," J. Acoust. Soc. Am., 80, pp. 955-960, 1986. 
[11] Shirai, T., Shibata, K., Takahashi, A., Mori, K., Kasashima, N., Ueno, Y., "Method for detecting faults in FFUs using SDP based on audio signal analysis," Proceedings of the IEEE Conference on Emerging Technologies and Factory Automation. ETFA '96, Kauai, Hawaii (USA), 1996.

[12] Shibata, K., Takahashi, A., Shirai, T., "Fault diagnosis of rotating machinery through visualization of sound signals," Mechanical Systems and Signal Processing, 14(2), pp. 229-241, 2000.

[13] Wu, J., Chuang, C., "Fault diagnosis of internal combustion engines using visual dot patterns of acoustic and vibration signals," NDT Int., 38, pp. 605-614, 2005.

[14] Bianchi, S., Corsini, A., Rispoli, F., and Sheard, A. G., "Detection of aerodynamic noise sources in low-speed axial fans with tip end-plates," Proc. Inst. Mech. Eng., Part C: J. Mech. Eng. Sci., 223, pp. 1379-1392, 2009.

[15] Bianchi, S., Corsini, A., Sheard, A., "Experiments on the use of signal visualization technique for in-service stall detection in industrial fans," Advances in Acoustics and Vibration, vol. 2013, ID610407, 10 pages.

[16] Xu, X., Liu, H., Zhu, H., Wang, S., "Fan fault diagnosis based on symmetrized dot pattern analysis and image matching," Journal of Sound and Vibration, vol. 374, pp. 297-311, 2016.

[17] Xu, X.G., Liu, H.X., Wang, S.L., Fan, Z.Y., Yang, L.J., Liu, S.T., "Real-time stall detection of centrifugal fan based on the analysis of symmetrized dot pattern and wavelet packet transform," Journal of VibroEngineering, vol. 19(3), pp. 1823-1832, 2017.

[18] ISO 5801:2007, "Industrial fans - Performance testing using standardized airways".

[19] Hameed, S., Samson, A., "Diagnosis of multiple faults of ball bearings using visual dot pattern", Proceedings of the 10th National Conference on Technological Trends (NCTT09), pp. 62-68, 2009.

[20] De Rosier, B., Normand, M. D., Peleg, M., "Effect of lag on the Symmetrized Dot Pattern (SDP) displays of the mechanical signatures of crunchy cereal foods," J. Sci. Food. Agric., vol. 75, pp. 173178, 1997. 
This document is a post-print version of the scientific paper published by Elsevier. It has been released by the authors to fulfill all the publisher requirements established for Article Sharing: https://www.elsevier.com/about/policies/sharing

\section{(9) $\odot \Theta \Theta$}

(C) 2019. This manuscript version is made available under the Creative Commons Attribution-NonCommercial-NoDerivatives 4.0 International License

(CC-BY-NC-ND 4.0 license) http://creativecommons.org/licenses/by-nc-nd/4.0/ 